To appear in Ecology

\title{
EVIDENCE FOR A GENERAL SPECIES-TIME-AREA RELATIONSHIP
}

Peter B. Adler ${ }^{1,6}$, Ethan P. White ${ }^{2, *}$, William K. Lauenroth ${ }^{3}$, Dawn M. Kaufman ${ }^{4}$, ANDREW RASSWEILER ${ }^{1}$, AND JAMES A. RUSAK ${ }^{5}$

${ }^{1}$ Department of Ecology, Evolution and Marine Biology, University of California, Santa Barbara, CA 93106.

${ }^{2}$ Department of Biology, University of New Mexico, Albuquerque, NM 87131.

${ }^{3}$ Department of Forest, Rangeland, and Watershed Stewardship, Colorado State University, Fort Collins, CO 80523 .

${ }^{4}$ Division of Biology, Kansas State University, Manhattan, KS 66506.

${ }^{5}$ Center for Limnology, University of Wisconsin, Trout Lake Station, Boulder Junction, WI 54512.

"Current address: Department of Biology, Utah State University, Logan, UT, 84321.

${ }^{6}$ E-mail: adler@lifesci.ucsb.edu

\begin{abstract}
The species-area relationship (SAR) plays a central role in biodiversity research, and recent work has increased awareness of its temporal analog, the species-time relationship (STR). Here we provide evidence for a general species-time-area-relationship (STAR), in which species number is a function of the area and time span of sampling, as well as their interaction. For eight assemblages ranging from lake zooplankton to desert rodents, this model outperformed a sampling-based model and two simpler models in which area and time had independent effects. In every case the interaction term was negative, meaning that rates of species accumulation in space decreased with the time span of sampling, while species accumulation rates in time decreased with area sampled. Although questions remain about its precise functional form, the STAR provides a tool for scaling species richness across time and space, for comparing the relative rates of species turnover in space and time at different scales of sampling, and for rigorous testing of mechanisms proposed to drive community dynamics. Our results show that the SAR and STR are not separate relationships but two dimensions of one unified pattern. Keywords: community dynamics, spatiotemporal scaling, species diversity, turnover, speciesarea relationship, species-time relationship

\section{INTRODUCTION}

The species-area relationship (SAR) provides a classic example of scale dependence: species richness depends on the size of the area sampled. The characteristic form of this relationship has motivated basic research (May 1975, Harner and Harper 1976, Shmida and Wilson 1985, Harte et al. 1999a) and proven valuable in conservation applications (Pimm and Askins 1995, Myers et al. 2000). Although first proposed by Preston (1960), only recently has considerable evidence been compiled for a temporal analogue of the SAR, the species-time relationship (STR; e.g. Rosenzweig 1995, Hadley and Maurer 2001, Adler and Lauenroth 2003, White 2004, White et al. in review). For the assemblages analyzed in these studies, species richness increased regularly with the time period of sampling.
\end{abstract}


Despite extensive work on the SAR, and a rapidly growing literature on the STR, we have yet to establish a firm understanding of how rates of species accumulation in space and time are related. Adler and Lauenroth (2003) took a first step in this direction by constructing a species-time-area-relationship (STAR) with data for tallgrass prairie plants in Kansas, modeling species number as a function of the area and time-span of sampling as well as their interaction. The interaction term was negative, meaning that the scaling exponent or "slope" of the STR decreased as the spatial scale of sampling increased, and similarly, the slope of the SAR decreased as the temporal period of observation increased. Equating species accumulation with species turnover (Harte and Kinzig 1997, Lennon et al. 2001), this means that the rate of spatial turnover decreases as the time span of sampling increases, and vice versa. Although sampling effects undoubtedly play some role in generating this pattern (more individuals are censused as spatial and temporal scales increase), sampling processes alone cannot explain the SAR (Rosenzweig 1995, Plotkin et al. 2000, Green et al. 2003) or the STR (Rosenzweig 1995, Adler and Lauenroth 2003, White 2004, White et al. in review), pointing to the importance of spatial and temporal heterogeneity in the distribution of species.

We believe the STAR has the potential to be an important tool in both applied and basic ecology. By showing how species number scales across time as well as space, and allowing comparison of rates of spatial and temporal turnover at different scales, it will improve biodiversity assessment and help researchers choose appropriate sampling scales. Furthermore, the STAR permits more rigorous testing of underlying processes than use of the SAR alone (Adler 2004, Maurer and McGill 2004). However, before this tool can be applied broadly, we must demonstrate its generality. We analyzed 8 datasets representing a variety of ecosystems and taxa to address three questions. First, does the general form of the STAR from the KS plant assemblage (Adler and Lauenroth 2003) hold for the additional datasets, or are simpler models superior? Second, at what sampling scales are rates of spatial and temporal turnover equivalent? Finally, can differences in methodology, environmental variability, or life-history explain variation among datasets in the values of the STAR parameters and relative rates of species turnover in space and time?

\section{Computational and statistical approach}

\section{METHODS}

A true STAR would require sampling at a series of spatial scales over many consecutive years. The datasets we collected provide continuous sampling through time, but in most cases sampled at only one spatial scale, such as the $10-\mathrm{m}^{2}$ plots for the KS plants. To represent increasingly large spatial scales, we aggregated these non-contiguous plots (details below). Such aggregation compresses environmental gradients and overestimates the influence of area on species number (Adler and Lauenroth 2003). Until multiscale, long-term data become available, this approach is the only way to explore the STAR.

After deciding on a method for spatial aggregation, we counted the number of species occurring in each spatial replicate, ranging from individual plots to aggregations of many plots, at a series of increasing time spans of observation. We used a temporal moving window approach, calculating species number in every possible one-year time span (20 one-year windows in a 20 year time series), then every possible time span of two consecutive years (19 windows), and so on, up to the time span of the entire time series (only one window). For every combination of area and time span sampled, we then calculated the mean species number (Adler and Lauenroth 2003). 
We fit these data to four alternative models. The first is a random sampling model, designed to test the null hypothesis that increases in species richness with time and area are simply a product of sampling more individuals at broader scales, and not a result of spatial and temporal heterogeneity in the distribution of species. This approach requires appropriate density data, which only two of our datasets (AZ rodents and KS small mammals) offered. To simulate random occurrence of individuals, we first created a species-abundance distribution (SAD) by summing the observed number of individuals of each species across the entire record. Next, we calculated the number of individuals occurring in each plot in each year. We then drew individuals at random without replacement from the SAD, filling up each plot in each year with the observed number of individuals. We repeated this simulation 500 times, then compared the simulated and observed means to calculate residual sums of squares and Akaike's Information Criteria (AIC), which penalizes models with extra parameters (Burnham and Anderson 1998).

The three other models represent power law based STARs of increasing complexity (Table 1). The "simplest model" treats species number as a power function of the product of time span and area sampled, with species number determined solely by this volume. This model assumes that time and area have equivalent and non-interactive effects on species number. The "no-interaction model" allows time and area to have different effects (species number can change at different rates for time span and area), but does not allow an interaction. As a result, STRs and SARs will not change with spatial or temporal scale, respectively (Rosenzweig 1998). The "full model," proposed by Adler and Lauenroth (2003), has parameters for time and area as well as a time-by-area interaction, allowing the effect of time span to vary with the spatial scale of sampling, and vice versa. For each dataset, we fit the models to the mean species number observed at each combination of time and area using linear regression on log-transformed variables (Table 1). Model parameters were similar when we used weighted linear regressions based on all individual observations (not shown). We compared the fit of the four candidate models using AIC. Because counts of species number at nested spatial and temporal scales are not independent, estimates of the standard errors of the regression parameters are invalid, preventing hypothesis testing. However, estimates of the regression parameters themselves are unbiased. The lack of independence does change the theoretical interpretation of AIC; in this case AIC serves only as an ad hoc penalty for the inclusion of additional parameters.

\section{Comparing species turnover in space and time}

Since species accumulation is equivalent to species turnover in many cases (Harte and Kinzig 1997, Harte et al. 1999b), one way to compare relative rates of species turnover in space and time is to compare the spatial and temporal scaling exponents of the STAR. If the full model best describes the data, however, the interaction term will complicate the interpretation of these exponents because temporal turnover will change as a function of spatial scale, and spatial turnover will change with temporal scale.

In order to compare rates of spatial and temporal turnover across multiple scales, we introduce the concept of "scales of time-area equivalence." The scales of equivalence are the particular combinations of sampled area and time-span at which measured rates of turnover in time and space will be equal. If we know the slope of a SAR based on one year of sampling, we can use the STAR to calculate the spatial scale that we would have to sample through time to produce an STR with the same slope. Solving the full model for these scales results in a constant proportional relationship between spatial and temporal scale (Appendix A). Therefore, these scales of equivalence are given by the ratio 


$$
A / T=10^{\left(\frac{z_{1}-w_{1}}{u}\right)}
$$

where $A$ is the area sampled to calculate a STR, $T$ is the time span sampled to produce a SAR, $z_{1}$ is the scaling exponent of the SAR at the unit time scale, $w_{1}$ is the scaling exponent of the STR at the unit spatial scale, and $u$ is the fitted interaction parameter. Any combination of sampling scales that correspond to this ratio will produce an STR and SAR with identical scaling exponents. For example, a ratio of $2-\mathrm{m}^{2} / 1-\mathrm{yr}$ means that the rate of temporal turnover measured on a $4-\mathrm{m}^{2}$ plot will equal the rate of spatial turnover calculated using data collected over a 2-year period.

\section{Dataset descriptions}

The KS plant composition dataset comes from tallgrass prairie at the Konza Prairie Biological Station, Kansas (Knapp et al. 1998), part of the Long-Term Ecological Research (LTER) network. We used 18 consecutive years of canopy cover data collected in $10-\mathrm{m}^{2}$ circular permanent plots. To represent larger sampling scales, we aggregated these plots by transect ( 5 plots), soil type (20 plots), watershed (40 plots), and study area (all 120 plots combined). Adler and Lauenroth (2003) provide more details on this approach, as well as results for a STAR based on natural log transformed mean species numbers. Here we re-fit the relationship using log base 10 and fit the three alternative models.

An additional dataset from the Konza LTER draws on 20 years of data for small mammals (rodents and insectivores; Kaufman et al. 2000). Twenty live-trapping stations are distributed systematically along each $285-\mathrm{m}$ line transect, with two traps set at each station for 4 nights each sampling period. We estimated that one transect samples an approximately 50x315$\mathrm{m}^{2}$ belt $\left(16,750-\mathrm{m}^{2}\right)$, with two transects located within each prescribed fire-grazing treatment. We used 14 permanent transects from seven treatments that were arranged in a relatively linear fashion, and aggregated at 4 levels: two transects within each treatment, all possible nearestneighbor pairs of treatments (4 transects), all possible nearest-neighbor triplets of treatments (6 transects), and all treatments (14 transects).

Long-term experiments in Chihuahuan desert scrub at Portal, AZ provide data on both rodents and vascular plants (Brown 1998). Treatments at Portal are assigned to $50 \times 50-\mathrm{m}^{2}$ plots. For both the rodents and plants, we used data from the 8 control plots. Rodent density is estimated by deploying a fixed-location grid of 49 traps within each plot one night each month (1978 through 2002). We represented larger spatial scales by aggregating the $50 \times 50-\mathrm{m}^{2}$ treatment plots as follows: pairs of nearest-neighbor plots ( 2 plots total), nearest quadruplets (4 plots), and all 8 plots combined. Two distinct plant guilds, corresponding to winter and summer annuals, have been censused for plant species composition on a grid of 16 permanent $0.25-\mathrm{m}^{2}$ quadrats within each $50 \times 50-\mathrm{m}^{2}$ treatment block (1988 through 2002). We aggregated these quadrats at 5 levels: groups of 4 neighboring individual quadrats, all 16 quadrats in each treatment plot, pairs of nearest-neighbor plots (32 quadrats), plot quadruplets (64 quadrats), and all quadrats at the site combined.

The intertidal algae and invertebrate datasets were collected at 3 sites along the central California coast (Schiel et al. 2004). Each site has two $30 \mathrm{~m}$ transects at different tidal elevations $\left(+0.3 \mathrm{~m}\right.$ and $+0.9 \mathrm{~m}$ mean lower low water). Within each tidal height, $101-\mathrm{m}^{2}$ permanent quadrats have been sampled annually for 27 years $(1976-2002)$, with all species present being recorded. We aggregated these data at 4 levels: half of each transect (5 individual quadrats), whole transects (10 quadrats), individual sites (20 quadrats), and all quadrats at all sites (60 quadrats). 
The Northern Temperate Lakes LTER site has monitored zooplankton assemblages in a series of lakes in Wisconsin for 21 years (Magnuson and Bowser 1990). For zooplankton species, five modified Schindler-Patalas trap samples per year ( 2 from the summer, 1 each from all other seasons) are identified and counted from one permanent station of maximum depth on each lake. We used pelagic species, removing all occurrences of littoral species. Arnott et al. (1998) showed that this sampling methodology effectively estimated whole lake pelagic zooplankton species richness. In contrast to the other datasets, we did not need to aggregate plots to represent increasingly large spatial scales. The lakes represent a range of sizes, so we could construct SARs for species numbers across lake size. Because the lakes are independent, these relationships will be more variable than the SARs based on non-independent, nested aggregations of plots.

\section{RESULTS}

Comparison of AIC values identified the full model, which included independent effects of time and area as well as an interaction term, as the best model for all eight datasets (Appendix B). The importance of including the interaction term is emphasized by the strong relationships between the time exponent and spatial scale, and between the area exponent and temporal scale (Fig. 1). The random sampling model, estimated only for the KS small mammals and AZ rodent datasets, was the worst model in both cases, with AIC values much less negative than even the simplest version of the STAR (Appendix B).

For the full model STARs based on mean species numbers, values of $z_{1}$, the slope of the linearized SAR given one year of sampling, ranged from 0.08 for NTL zooplankton to 0.42 for AZ summer annuals (Table 2, mean species numbers shown in Appendix C). Except for the zooplankton, all values were greater than 0.22 . Values of $w_{1}$, the slope of the STR given a spatial sample of $1-\mathrm{m}^{2}$, were higher, ranging from 0.20 (CA intertidal algae) to 1.0 (KS small mammals). The other six datasets had values of $w_{1}$ between 0.35 and 0.50 . All interaction parameters were negative (Fig. 1): values ranged from -0.02 for intertidal algae to -0.18 for AZ summer annuals (Table 2).

From these STARs, we calculated the ratios that specify the scales of time-area equivalence, pairs of spatial and temporal sampling scales at which STRs and SARs, respectively, have equal slopes. The ratios spanned 12 orders of magnitude. CA intertidal algae had ratios of equivalence of $0.05-\mathrm{m}^{2} / 1-\mathrm{yr}^{1}$, the only value less than $1-\mathrm{m}^{2} / 1-\mathrm{yr}^{1}$. CA intertidal invertebrates, and both groups of $\mathrm{AZ}$ annual plants had values between 2 and $6-\mathrm{m}^{2} / 1-\mathrm{yr}^{1}, \mathrm{KS}$ plants reached equivalence at about 50 (Fig. 2), AZ rodents at 8,000, and KS small mammals at $14,000-\mathrm{m}^{2} / \mathrm{yr}$, whereas NTL zooplankton did not reach equivalence until 460,000-ha/yr (Table 2).

\section{Evidence for a general STAR}

\section{DisCUSSION}

The 7 new STARs that we constructed for taxa ranging from lake zooplankton to desert rodents were all qualitatively consistent with the previously published STAR for KS tallgrass plants (Adler and Lauenroth 2003). In every case, a model with independent parameters for time-span, area, and an interaction term was superior to simpler models. The time-by-area interaction was always negative, meaning that the slope of the SAR decreased as the temporal scale of sampling increased, and the slope of the STR decreased as area sampled increased (Fig. 1). If one of the simpler models, which contained no interaction term, had provided the best fit, it would suggest that time and area have independent effects on species number. The existence of 
a strong time-by-area interaction indicates that SARs and STRs represent two axes of one unified STAR, and suggests that similar underlying processes may operate in space and time.

While the full interaction models outperformed simpler models, a number of questions remain about the specific functional form of the STAR. Both power law and logarithmic forms of the model give good fits for most datasets (not shown), consistent with the results of analyses for STRs (Rosenzweig 1995, White 2004, White et al. in review) and for SARs (Connor and McCoy 1979, Lennon et al. 2001). More importantly, our STAR model assumes a linear relationship between $w$ and log area, and between $z$ and log time-span. In the extreme we know this to be incorrect because it would allow $w$ and $z$ to take negative values at very broad spatial or temporal scales. As more appropriate data become available, future research should address such questions about the precise functional form of the STAR.

Rates of species turnover in space and time

The simplest way to compare relative rates of species accumulation, or turnover, in space and time is to compare the values of the time slope, $w_{l}$, and the area slope, $z_{l}$. In every dataset except the intertidal algae, $w_{1}$ was greater than $z_{1}$, meaning that for a $1-\mathrm{m}^{2}$ sample, increasing the time span of observation from 1 to 10 years resulted in the addition of more new species than increasing the area of sampling from 1 to $10-\mathrm{m}^{2}$. This is a critical result, since much field ecology is conducted at these fine scales, often with little consideration of temporal variability.

Under the full STAR model, this simple comparison of $w_{l}$ and $\mathrm{z}_{1}$ is complicated by the interaction term. To compare rates of spatial and temporal turnover at multiple scales, we estimated the sampling scales at which spatial and temporal turnover are equal. For the AZ annuals and intertidal invertebrates, the ratio determining these scales of time-area equivalence was in the single digits, meaning that spatial turnover collected on the scale of years is very similar to temporal turnover collected on the scale of meters. For the KS plants, the ratio was an order of magnitude larger (approximately $50-\mathrm{m}^{2} / \mathrm{yr}^{1}$ ), so for every year sampled while estimating spatial turnover, we must repeatedly sample $50-\mathrm{m}^{2}$ to find a similar rate of temporal turnover (illustrated in Fig. 2). The small mammals had ratios on the order of $10^{3}$ or approximately 1 ha/yr and the zooplankton had ratios several orders of magnitude larger. Three potential sources of this variability are methodological differences among datasets, differences in the environmental variability of each ecosystem, and contrasts in life history of the focal taxa.

Explaining variability among datasets

Methodological differences among our datasets create one source of variability in parameter values and scales of equivalence. Sampling methods for the plant and intertidal datasets are similar, based on fixed quadrats of similar size. The small mammal datasets, in contrast, rely on trapping, making determination of area sampled more uncertain. However, the most important methodological difference concerns the zooplankton data, for which broader scales correspond to larger whole lakes, analogous to an SAR for islands, while in other datasets we represented broader scales by aggregating subplots. The zooplankton's low $z_{1}$, which in turn contributes to a high ratio of equivalence, may reflect this difference.

A more interesting explanation of variability among datasets invokes ecosystem differences in patterns of environmental variability. The expectation is that variability in species composition is associated with variability in the environment. For example, the larger ratio for KS plants compared to AZ desert plants may reflect the greater fine scale spatial heterogeneity in the desert, where bare ground is extensive and plant cover patchy. Differences in temporal variability may also be important. In seasonally variable environments, such as deserts and grasslands, a key environmental variable such as soil moisture might vary more from year-to- 
year than from one $1-\mathrm{m}^{2}$ plot to another during one year. The marine environment represented by the intertidal datasets, in contrast, may be more stable in time, but highly variable in space due to the importance of depth. This difference could explain why the intertidal algae had a much lower $w_{l}$ and ratio of equivalence than the desert and grassland plants.

Life history differences offer an intriguing but poorly understood source of variability. Unlike the slope of the power-law SAR or STR (Rosenzweig 1995), $w_{1}$ and $z_{1}$ in the full model of the STAR are inherently dependent on the choice of units. Therefore, we should expect organisms operating at different characteristic scales to exhibit different scales of equivalence. For example, while $1-\mathrm{m}^{2}$ may contain many individuals and species of herbaceous plants, for small mammals, which have much larger home ranges and lower population density, a $1-\mathrm{m}^{2}$ sample means something quite different. From this perspective, many of the differences among datasets in the ratios of equivalence (Table 2) appear to make intuitive sense: ratios were much larger for the mobile vertebrates and zooplankton than for plants, algae, or intertidal invertebrates, presumably because the vertebrates and zooplankton are more mobile or widely dispersed, and sample the spatial environment at a coarser resolution.

If we wished to remove these life history influences, one possibility would be to rescale area by home range size. Re-fitting the STAR model to the KS small mammals data using a spatial unit of $0.5 \mathrm{ha}$, a reasonable estimate of average home range size for this species assemblage, gives a $w_{l}$ of 0.40 and a $z_{l}$ of 0.33 , similar to the values from plant datasets, and the ratio of equivalence falls from $14,000-\mathrm{m}^{2} \cdot \mathrm{yr}^{-1}$ to 2.9 "home range units" per yr. Rescaling the AZ small mammals by the same factor results in a similar rescaled ratio of 1.6. Similar rescaling in time would be possible based on traits such as generation time, perhaps standardizing comparisons of organisms as disparate as annual plants and forest trees. However, rescaling will only be appropriate to the extent that life history traits among species within a community are homogeneous, and it is unclear how rescaling could be applied to taxonomic groups without easily defined home ranges. More importantly, appealing as these rescaling exercises may be, their utility will be limited until we gain a theoretical understanding of how life history traits should influence the parameters of the STAR. We see great potential for modeling to generate testable hypotheses about the influence of traits such as mobility and longevity or dispersal and dormancy. Neutral models may provide an appropriate framework, as they have already demonstrated the influence of dispersal on the slope of the SAR in a homogeneous environment (Bell 2001, Hubbell 2001).

\section{Conclusion}

The general pattern described by the STAR is not the product of spatiotemporal environmental variation alone, but of demographic processes and ecological interactions played out on a template of environmental variation (Storch et al. 2003, Tuomisto et al. 2003). Teasing apart the role of these different factors and processes in generating macroscopic patterns is a central but stubborn problem in ecology (Levin 1992). We are optimistic that quantifying rates of spatial and temporal turnover at different scales will improve our ability to identify the most important underlying processes (e.g. Adler 2004).

The most important result from this and earlier work (Adler and Lauenroth 2003) is the recognition that the SAR and STR are not independent relationships but rather two components of a single species-time-area relationship (STAR). The parameters of the STAR allow scaling of species number across space and time as well as calculation of scales of time-area equivalence, the sampling scales at which spatial and temporal rates of species turnover are equal. The 
STAR, along with derived metrics such as the scales of equivalence, offers an essential tool for understanding, describing, and comparing the spatiotemporal dynamics of ecological systems.

Acknowledgements

The following individuals and institutions generously contributed data: J. H. Brown, D. W. Kaufman, G. A. Kaufman, J. R. Steinbeck, Pacific Gas \& Electric Co., and the Portal LTREB. Data collection at the Konza Prairie and North Temperate Lakes LTER sites was supported by the NSF Long-Term Ecological Research Program, which also funded our "Species richness in space and time" working group. We thank the members of this group for valuable discussion and M. Smith, J. Yao, and S.P. Carey for comments on an earlier draft. PBA was supported by a NSF Post-doctoral fellowship in Biological Informatics and EPW was supported by an NSF Graduate Research Fellowship.

\section{LITERATURE CITED}

Adler, P. B. 2004. Neutral models fail to reproduce observed species-time and species-area relationships in Kansas grasslands. Ecology 85:1265-1272.

Adler, P. B., and W. K. Lauenroth. 2003. The power of time: spatiotemporal scaling of species diversity. Ecology Letters 6: 749-756.

Arnott, S. E., J. J. Magnuson, and N. D. Yan. 1998. Crustacean zooplankton species richness: single- and multipleyear estimates. Canadian Journal of Fisheries and Aquatic Sciences 55: 1573-1582.

Brown, J. H. 1998. The Desert Granivory Experiments at Portal, Pages 71-95 in W. J. J. Resetarits, and J. Bernardo, eds. Experimental Ecology. New York, USA. Oxford University Press, Oxford, England.

Burnham, K. P., and D. R. Anderson. 1998. Model selection and inference: a practical information-theoretic approach. Springer, New York.

Connor, E. F. and McCoy, E. D. 1979. Statistics and biology of the species-area relationship. American Naturalist 113: 791-833.

Green, J., J. Harte, and A. Ostling. 2003. Species richness, endemism and abundance patterns: tests of two fractal models in a serpentine grassland. Ecology Letters 6: 919-928.

Hadley, E. A., and B. A. Maurer. 2001. Spatial and temporal patterns of species diversity in montane mammal communities of western North America. Evolutionary Ecology Research 3: 477-486.

Harner, R. F., and K. T. Harper. 1976. The role of area, heterogeneity and favorability in plant species diversity of pinyon-juniper ecosystems. Ecology 57: 1254-1263.

Harte, J, Kinzig, A, and Green J. 1999a. Self-similarity in the distribution and abundance of species. Science 284: 334-336.

Harte, J. and Kinzig, A. P. 1997. On the implications of species-area relationships for endemism, spatial turnover, and food web patterns. Oikos 80: 417-427.

Harte, J., S. McCarthy, K. Taylor, A. Kinzig, and M. L. Fischer. 1999b. Esimating species-area relationships from plot to landscape scale using species spatial-turnover data. Oikos 86: 45-54.

Kaufman, D. M., G. A. Kaufman, and D. W. Kaufman. 2000. Faunal structure of small mammals in tallgrass prairie: an evaluation of richness and spatiotemporal nestedness. Pages 47-70 in J. R. Choate, editor. Reflections of a naturalist: papers honoring Professor Eugene D. Fleharty, Fort Hays Studies, Special Issue, 1:1-241.

Knapp, A. K., Briggs, J. M., Hartnett, D. C. and S. L., Collins (eds.). 1998. Grassland dynamics: long-term ecological research in tallgrass prairie. Oxford University Press, Oxford.

Lennon, J. J., Koleff, P., Greenwood, J. J. D. and Gaston, K. J. 2001. The geographical structure of British bird distributions: diversity, spatial turnover and scale. Journal of Animal Ecology 70: 966-979.

Levin, S. A. 1992. The problem of pattern and scale in ecology. Ecology 73:1943-1967.

Maurer, B. A., and B. J. McGill. Neutral and non-neutral macroecology. Basic and Applied Ecology 5: 413-422.

May, R. M. 1975. Patterns of species abundance and diversity. Ecology and Evolution of Communities. eds. M. L. Diamond J. M. Cody, 81-120. Belknap Press, Cambridge, MA.

Magnuson, J. J., and C. J. Bowser. 1990. A network for long-term ecological research in the United States. Freshwater Biology 23:137-43.

Myers, N., R. A. Mittermeier, C. G. Mittermeier, G. A. B. da Fonseca, and J. Kent. 2000. Biodiversity hotspots for conservation priorities. Nature 403: 853-858. 
Pimm, S. L., and R. A. Askins. 1995. Forest losses predict bird extinctions in eastern North America. Proceedings of the National Academy of Sciences 92: 9343-9347.

Plotkin, J. B., M. D. Potts, N. Leslie, N. Manokaran, J. LaFrankie, and P. S. Ashton. 2000. Species-area curves, spatial aggregation, and habitat specialization in tropical forests. Journal of Theoretical Biology 207: 81-99.

Preston, F. W. 1960. Time and space and variation of species. Ecology 41: 611-627.

Rosenzweig, M. L. 1995. Species Diversity in Space and Time. Cambridge University Press, Cambridge, England.

Rosenzweig, M. L. 1998. Preston's ergodic conjecture: the accumulation of species in space and time. Biodiversity dynamics: turnover of populations, taxa, and communities. Eds M. L. McKinney, and J. A. Drake, 311-348. Columbia University Press, New York.

Schiel, D. R., J. R. Steinbeck, and M.S. Foster. 2004. Ten years of induced ocean warming causes comprehensive changes in marine benthic communities. Ecology 85: 1833-1839.

Shmida, A., and M. V. Wilson. 1985. Biological determinants of species diversity. Journal of Biogeography 12: 120.

Storch, D., A. L. Šzling, and K. J. Gaston. 2003. Geometry of the species-area relationship in central European birds: testing the mechanism. Journal of Animal Ecology 72: 509-519.

Tuomisto, H., K. Ruokolainen, and M. Yli-Halla. 2003. Dispersal, environment, and floristic variation of western Amazonian forests. Science 299: 241-244.

White, E. P. 2004. Two-phase species-time relationships in North American land birds. Ecology Letters 7: 329-336.

White, E. P., P. B. Adler, W. K. Lauenroth, R. A. Gill, D. Greenberg, D. M. Kaufman, A. Rassweiler, J. A. Rusak, M. D. Smith, J. R. Steinbeck, R. B. Waide, and J. Yao. A comparison of the species-time relationship across ecosystems and taxonomic groups. Oikos (in review).

Table 1. Alternative models for the species-time-area relationship (STAR). $S$ is species number, $A$ is area, $T$ is time span, $z$ is the scaling exponent or "slope" of the SAR, $w$ is the slope of the STR, $u$ is the interaction parameter, and $c$ is a constant. In the full model, we use $z_{l}$ rather than $z$, the traditional, time-independent SAR slope, to emphasize that this is the slope of the SAR calculated using a temporal scale of one-year; similarly, $w_{l}$ is the slope of the STR given a spatial scale of $1-\mathrm{m}^{2}$. Data were fit to the log-transformed models (log base 10).

\begin{tabular}{lll}
\hline Description & Power law form & $\log$ transformation \\
\hline $\begin{array}{l}\text { Simplest model } \\
\text { No interaction }\end{array}$ & $S=c(A T)^{z}$ & $\log S=c+z \log (A T)$ \\
model & $S=c A^{z} T^{w}$ & $\log S=c+z \log A+w \log T$ \\
Full model & $S=c A^{\left(z_{1}+\frac{u}{2} \log T\right)} T^{\left(w_{1}+\frac{u}{2} \log A\right)}$ & $\log S=c+z_{1} \log A+w_{1} \log T+u \log A \log T$ \\
\hline
\end{tabular}

Table 2. Parameters for the full model of the species-time-area relationship, fit to mean species number at each spatial and temporal scale of sampling. The $\mathrm{R}^{2}$ shows percent of the variation in mean species number at each area and time span explained by the model; the model does not explain variation in species number among replicate observations within each scale of sampling. The "Ratio of scales of equivalence" specifies combinations of sampling scales at which the slope of the species-time and species-area relationships are equal, and has units $\mathrm{m}^{2} \cdot \mathrm{yr}^{-1}$.

\begin{tabular}{|c|c|c|c|c|c|c|}
\hline Dataset & $c$ & $z_{1}$ & $w_{1}$ & $u$ & $\begin{array}{c}\text { Multiple } \\
\mathrm{R}^{2}\end{array}$ & $\begin{array}{l}\text { Ratio of scales of } \\
\text { equivalence }\end{array}$ \\
\hline KS plants & 1.169 & 0.273 & 0.370 & -0.056 & 0.977 & 52 \\
\hline AZ winter annual plants & 1.031 & 0.324 & 0.423 & -0.134 & 0.971 & 5.5 \\
\hline AZ summer annual plants & 0.865 & 0.425 & 0.495 & -0.178 & 0.983 & 2.5 \\
\hline Intertidal (CA) algae & 1.120 & 0.235 & 0.204 & -0.024 & 0.966 & 0.05 \\
\hline Intertidal (CA) invertebrates & 0.991 & 0.309 & 0.353 & -0.072 & 0.961 & 4.1 \\
\hline KS small mammals & -0.792 & 0.330 & 1.004 & -0.162 & 0.970 & 14,000 \\
\hline AZ rodents & 0.120 & 0.215 & 0.374 & -0.041 & 0.989 & 8,000 \\
\hline NTL zooplankton & 0.989 & 0.084 & 0.489 & -0.042 & 0.919 & $4.6 \times 10^{9}$ \\
\hline
\end{tabular}



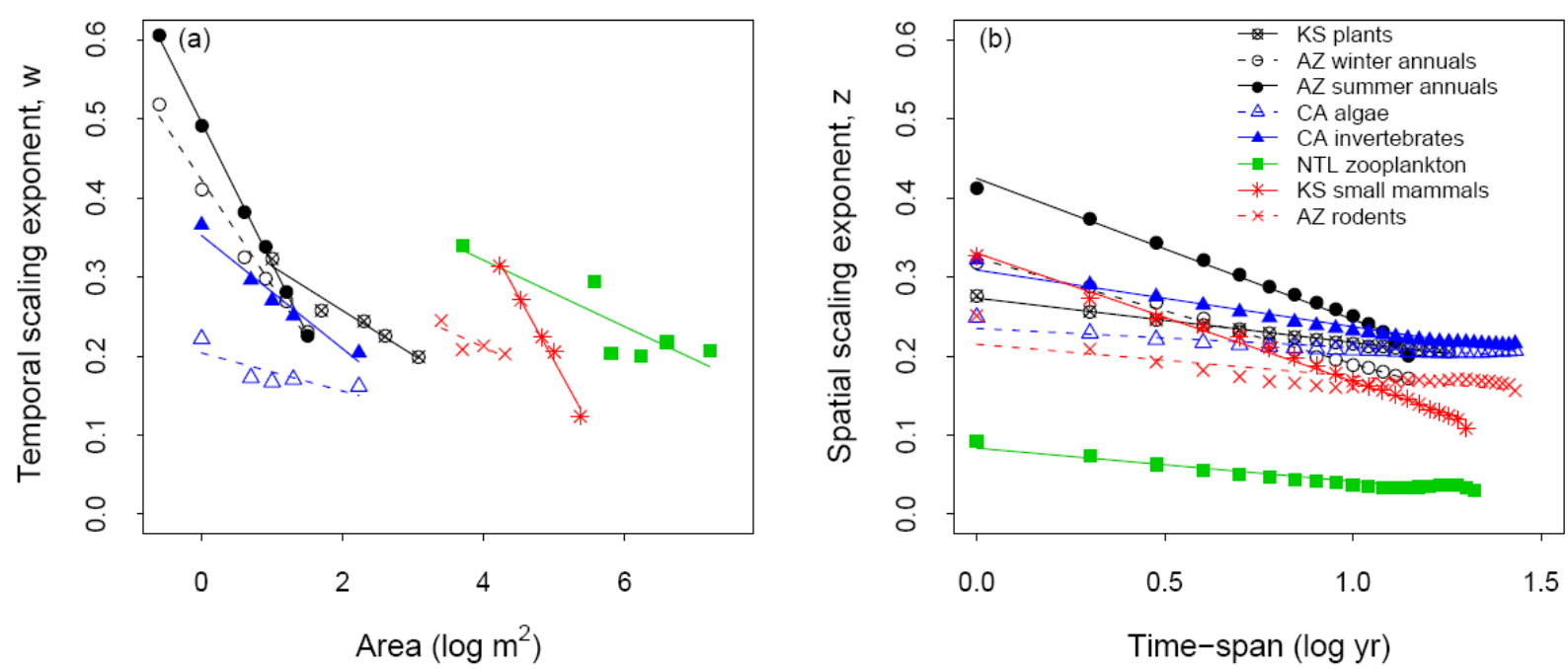

FIG. 1. (a) Decreases in $w$, the slope of the species-time relationship (STR), with increasing spatial scale of sampling. Symbols give the value of $w$ produced by subsetting the full dataset for a given value of area sampled, then regressing $\log$ species number on $\log$ time-span. (b) Decreases in $z$, the slope of the species-area relationship, with increases in the temporal scale of sampling. Symbols give the value of $z$ produced by subsetting the full dataset for a given value of time-span, then regressing log species number on log area. In both panels, the dashed lines running through each set of points show $w$ and $z$ predicted by the full interaction form of the STAR (formula in Table 1, parameters in Table 2).

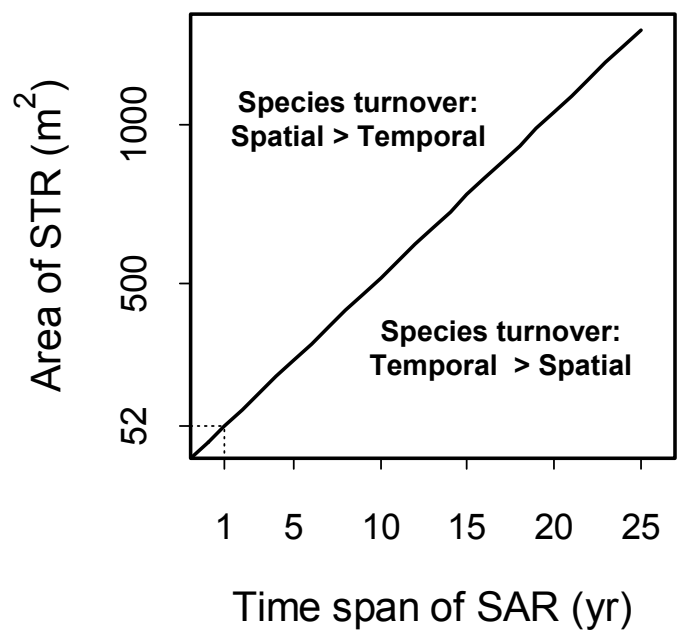

FIG. 2. Sampling scales that yield equivalent rates of species accumulation in space and time for the Konza plant community. The x-axis shows the time span sampled for a species-area relationship (SAR), while the y-axis shows the area sampled for a species-time relationship (STR). The line shows combinations of scales that produce SARs and STRs with identical scaling exponents. Below the line, the STR will have a steeper slope than the SAR, indicating higher relative turnover in time than in space. Above the line, the opposite occurs. The slope of the line (52 in this case), which also can be represented as a ratio (see Table 2), determines the "scales of timearea equivalence" for this dataset. 